\title{
Lossless polarization attraction of copropagating beams in telecom fibers
}

\author{
Victor V. Kozlov, ${ }^{1}$ Matteo Barozzi, ${ }^{2, *}$ Armando Vannucci, ${ }^{2}$ and Stefan Wabnitz ${ }^{3}$ \\ ${ }^{1}$ Department of Physics, St. Petersburg State University, Petrodvoretz, St. Petersburg 198504, Russia \\ ${ }^{2}$ Dipartimento di Ingegneria Dell'Informazione, Università degli Studi di Parma Viale delle Scienze 181/A, \\ Parma 43124, Italy \\ ${ }^{3}$ Department of Information Engineering, Università di Brescia, Via Branze 38, Brescia 25123, Italy \\ ${ }^{*}$ Corresponding author: matteo.barozzi82@gmail.com
}

Received November 1, 2012; revised December 18, 2012; accepted December 26, 2012; posted January 4, 2013 (Doc. ID 179084); published February 12, 2013

\begin{abstract}
We study the performance of a nonlinear lossless polarizer (NLP), the device that transforms an input arbitrary state of polarization (SOP) of a signal beam into one and the same SOP toward the output and, unlike conventional passive polarizers, performs this transformation without polarization-dependent losses. The operation principle of this device is based on the nonlinear rotation of the SOP of the strong signal beam under the interaction with a copropagating strong pump beam in a Kerr medium, which in our case is a telecom fiber. We quantify the performance of this NLP by introducing the notion of instantaneous degree of polarization, which is a natural extension of the conventional notion of the degree of polarization appropriate for CW beams to the case of pulses whose SOP is not constant across the pulse. We pay particular attention to the regime when signal and pump beams experience a walk-off in the dispersive medium. In particular, we demonstrate that a signal pulse experiences much stronger repolarization when the walk-off effect is present as compared with the case of no walk-off. We also study the degradation of the efficiency of the NLP in the presence of polarization mode dispersion. (c) 2013 Optical Society of America
\end{abstract}

OCIS codes: $\quad 230.5440,060.4370,230.1150,230.4320$.

\section{INTRODUCTION}

To the best of our knowledge, the history of nonlinear lossless polarizers (NLPs) started from the proposal of Heebner et al. [1], where it was experimentally demonstrated that the effect of two-wave mixing in a photorefractive material could be used for the amplification of one polarization component of a light beam by using the orthogonal component as a pump beam. The major advantage of this technique is that the energy of the beam is conserved in the repolarization process, while the use of a linear passive polarizer inevitably brings (on average) a waste of $50 \%$ of the beam energy. Due to this conservation property, the NLP preserves the intensity of the outcoming beam, even if the state of polarization (SOP) of the incoming beam changes with time. In contrast, the use of a linear passive polarizer introduces polarization-dependent losses; therefore, the outcoming beam suffers from intensity fluctuations. These fluctuations can be undesirable for the operation of other devices (e.g., a parametric amplifier) that may follow the polarizer. The NLP can become the right choice in such optical schemes. Moreover, NLPs offer a light-by-light control over the SOP of the signal beam, which provides a more robust, reliable, and potentially faster technology than what is available with mechanically or electrically controlled systems. With an eye toward fast optical control of the SOP, slow photorefractive materials should be changed to media with a faster nonlinear response. The immediate candidate is optical fibers with their virtually instantaneous Kerr nonlinearity. Indeed, NLPs based on the interaction of copropagating strong signal and pump beams in a span of optical fiber offer, at least in principle, the possibility to control the signal SOP on the scale of a few femtoseconds [2]. It is this type of NLP that is the object of the present study.

Previous studies anticipating the fiber-based NLP date back to 1998 , where the model equations for the (isotropic) fiberbased NLP were used for predicting the polarization switching of the signal beam induced by a counterpropagating quasi-CW pump, a result supported by its full experimental confirmation $[3,4]$. In the experiments which followed, the polarization attraction effect lying at the heart of any NLP was experimentally observed in short (i.e., meter-long) samples of spun isotropic [5, 6 ] or bimodal fiber [7], and in a practical, kilometer-long span of telecom fiber [8], eventually leading to the development of an all-optical regenerator of both the SOP and the intensity profile of modulated telecom signals [9]. This impressive experimental progress has been accompanied by simultaneous developments on the theoretical side. Theories of polarization attraction have been developed for isotropic fibers [7,10-13], for telecom fibers [14,15], and for unidirectionally spun and high-birefringence fibers $[\underline{15}, 16]$.

All of the activities we listed in the preceeding paragraph are related to the counterpropagation geometry, where signal and pump beams (approximately of equal powers) propagate in opposite directions. In this geometry, the two beams require some transient time to establish a joint equilibrium state, which is called the polarization attractor. It is this state that is associated with the repolarization (or polarization attraction) of the signal beam from its input to its output at the end of the fiber. How long is this transient time? This question has been asked and answered in a recent paper [17], both theoretically as well as experimentally. For parameters that are 
relevant to telecom fibers and power levels around $1 \mathrm{~W}$, the transient time does not drop below $1 \mu \mathrm{s}$. Therefore, a signal beam whose SOP is changing in time faster than $1 \mu$ s cannot be repolarized. This time scale is significantly faster not only than the response time of photorefractive materials, which is seconds or minutes, but also of the $1 \mathrm{~ms}$ to $150 \mu$ s response time of commercial electro-optical polarization controllers, which are based on electronic feedback. Nevertheless, the counterpropagating scheme appears to be not capable of fully realizing the potential of femtosecond-scale response, which is inherent to the Kerr nonlinearity in optical fibers. The way out has been proposed in [2], where it was theoretically shown that an NLP may also be constructed by using signal and pump beams in the copropagating geometry. Indeed, the pioneering work [18] had numerically demonstrated a polarization attraction effect in copropagation (there called "polarization funnel"), limited to the case of circular polarizations and occurring in perfectly isotropic fibers. The response of an NLP in the copropagating geometry is, at least in principle, as fast as the Kerr response of the medium. Thus, such a polarizer could be readily applied to telecommunications and the processing of ultrafast signals, even in the extreme cases where the SOP of a bit stream varies significantly across each individual pulse.

Regarding the comparison between the co- and counterpropagating configurations, it should be noted that if the pump and signal beams copropagate at different group velocities, the two beams can be seen as counterpropagating waves in a frame moving at their average group velocity [18]. What makes the difference, under such a unified viewpoint, is the relative pump-signal velocity, which is enormously large (in the order of the speed of light) in counterpropagation, whereas it may even vanish in the copropagation of groupvelocity-matched pump and signal waves [2]. The theoretical consideration of [2] was the demonstration of the proof of principle of a copropagating NLP, and it was mainly oriented to the case of $\mathrm{CW}$ beams. The main goal of the present paper is to generalize the theory of this device to the practical case when the signal beam consists of a train of picosecond pulses. Such a task is not trivial. The problem is that the repolarization (attraction) process is a sensitive function of (both pump and signal) power. Therefore, different parts of the same pulse experience different degrees of repolarization (DOPs); as a result, the SOP of the outcoming signal is not uniform across each individual pulse. An open problem is also how one can apply the notion of DOP to describe such a pulse with complex polarization dynamics. Already from these preliminary considerations it becomes clear that the DOP of a repolarized pulse is always lower than the DOP of a CW beam with the same value of peak intensity. Given that the upper limit to the DOP of a CW signal out of a single copropagating NLP is already not very high, namely only 0.73 [2]; methods to improve the performance of the codirection NLP with a pulsed signal are greatly needed. Here we also solve this problem by means of a dispersive fiber, so that a substantial walk-off appears between the signal pulse and the CW pump beam through the length of the fiber. As noted above, this situation may be viewed as a counterpropagating configuration, albeit with a very limited and precisely controlled amount of pumpsignal mutual velocity. Hence, the configuration of our interest lies in between the extreme cases of vanishing walk-off [2] and maximal walk-off (counterpropagation) [17], both of which exhibit poor performances in the repolarization of relatively short (picosecond) signal pulses. All of these problems are addressed and solved in the present paper, which thereby paves the way to the experimental demonstration of an efficient copropagating NLP.

\section{MODEL}

In this paper we consider the polarization interaction in an optical fiber of a CW pump beam and a signal beam consisting of a stream of short optical pulses. Both beams are supposed to be copropagating along the $z$ axis through a (lossy) fiber of total length $L$. We also assume that the optical pulses are sufficiently spaced from each other so that only one pulse interacts with each time sample of the pump along the fiber span. With this simplification, we can concentrate our attention on the interaction dynamics of an intensive $\mathrm{CW}$ pump beam with a single pulse from the signal stream, thus avoiding complications associated with interpulse interactions mediated by the presence of the pump beam.

As a propagation medium, we consider the practically relevant case of a randomly birefringent (telecom) fiber. If the randomness of the fiber birefringence is taken into account through the Manakov equation $[\underline{2}, \underline{14}, \underline{19}]$, the evolution equations for the (unattenuated) Stokes vectors of the signal and pump beams, $\mathbf{S}^{(s)}=\left(S_{1}^{(s)}, S_{2}^{(s)}, S_{3}^{(s)}\right)^{T}$ and $\mathbf{S}^{(p)}=$ $\left(S_{1}^{(p)}, S_{2}^{(p)}, S_{3}^{(p)}\right)^{T}$ (we adopt here the Stokes elements numbering for which $S_{3}$ is the circular polarization), are

$$
\begin{gathered}
\left(\partial_{z}-v_{s} \partial_{t}\right) \mathbf{S}^{(s)}=\gamma e^{-\alpha z} \mathbf{S}^{(s)} \times \mathbf{J}_{x}(z) \mathbf{S}^{(p)}, \\
\left(\partial_{z}-v_{p} \partial_{t}\right) \mathbf{S}^{(p)}=\gamma e^{-\alpha z} \mathbf{S}^{(p)} \times \mathbf{J}_{x}(z) \mathbf{S}^{(s)},
\end{gathered}
$$

where $\alpha=0.2 \mathrm{~dB} / \mathrm{km}$ is the fiber scattering loss [which should be applied to $\mathbf{S}^{(s, p)}$ after solving Eqs. (1) and (2)], $\gamma$ is the fiber nonlinear coefficient, and $v_{s, p}$ are group velocities at the signal $\omega_{s}$ and pump $\omega_{p}$ carrier frequencies, respectively. Moreover, $\times$ denotes cross product, and $\mathbf{J}_{x}(z)=$ $\operatorname{diag}\left(J_{x 1}(z), J_{x 2}(z), J_{x 3}(z)\right)$ is a cross-polarization tensor, whose $z$-varying diagonal components can be found from a set of linear ordinary differential equations with constant coefficients as discussed in $[\underline{14}, \underline{19}]$. The cross-polarization tensor coefficients depend on the magnitudes of the birefringence $\Delta \beta\left(\omega_{s, p}\right)$ at $\omega_{s}$ and $\omega_{p}$, as well as on the birefringence correlation length $L_{c}$. The randomly birefringent fiber is characterized by its polarization mode dispersion (PMD) coefficient $D_{p}=2 \sqrt{2} \pi \sqrt{L_{c}} /\left[L_{B}\left(\omega_{s}\right) \omega_{s}\right]$, where $L_{B}\left(\omega_{s}\right)=2 \pi / \Delta \beta\left(\omega_{s}\right)$ is the beat length at $\omega_{s}$. Another important length scale in the problem is the PMD diffusion length $L_{d}$, which is defined as $L_{d}^{-1}=1 / 3 D_{p}^{2}\left(\omega_{p}-\omega_{s}\right)^{2}$. Equations (1) and (2) are strictly valid in two limits: $L, L_{\mathrm{NL}} \ll L_{d}$, which we call here the Manakov limit, and $L, L_{\mathrm{NL}} \gg L_{d}$, which is named the diffusion limit. As $\mathbf{J}_{x}(z)=\operatorname{diag}(0,0,0)$ in the diffusion limit, such case is not interesting to us because the polarization mixing that is vitally important for the operation of the NLP is absent. In the Manakov limit, where we restore the vector Manakov equation without the group-velocity dispersion (GVD) terms [20], we get $\mathbf{J}_{x}=-8 / 9 \operatorname{diag}(1,1,1)$. In this case the NLP operates most efficiently; therefore, this limit is of primary interest to us. As shown in [2], the deviation of the parameter regime away 
from the Manakov limit leads to degradation of the performance of the NLP. This observation defines our choice to work in the Manakov limit, in which case Eqs. (1) and (2) simplify to

$$
\begin{gathered}
\left(\partial_{z}-v_{s} \partial_{t}\right) \mathbf{S}^{(s)}=\bar{\gamma} e^{-\alpha z} \mathbf{S}^{(p)} \times \mathbf{S}^{(s)}, \\
\left(\partial_{z}-v_{p} \partial_{t}\right) \mathbf{S}^{(p)}=\bar{\gamma} e^{-\alpha z} \mathbf{S}^{(s)} \times \mathbf{S}^{(p)},
\end{gathered}
$$

where $\bar{\gamma}=(8 / 9) \gamma$. In [2] these equations have been solved analytically for the case of no walk-off: $v_{s}=v_{p}$. Here we add the walk-off terms; thus we convert the ordinary differential equations of [2] into the partial differential equations of Eqs. (3) and (4), which are to be solved numerically.

Equations (3) and (4) are valid in the limit of zero PMD. Because our interest is also in considering the case of relatively high PMD fibers, we numerically solved the Manakov-PMD propagation equation [21], by using Optilux [22], an open-source simulator, based on the split-step Fourier method. Within Optilux, the random birefringence of the fiber was rigorously taken into account by using a correlation length $L_{c}=10 \mathrm{~m}$, so that each fiber was modelled by means of hundreds of birefringent waveplates. Although all numerical results in this paper were obtained from Optilux, in the case of negligible PMD and zero chromatic dispersion we performed a cross check with the direct solution of Eqs. (3) and (4), and we confirmed that the two methods produce almost identical results. This validates the simple and numerically fast simulation approach which is based on Eqs. (3) and (4) as a useful tool for modeling zero- and low-PMD waveguides.

In our present study we consider a parameter regime that is similar to the case of experiments with counterpropagating NLPs [8,14,17]; namely, we fix the fiber nonlinear coefficient $\gamma=1(\mathrm{~W} \cdot \mathrm{km})^{-1}$, the signal peak pulse power $S_{0}^{(s)}=1 \mathrm{~W}$, and the Gaussian signal pulse FWHM $=12.5$ ps. Note that the nonlinear length is defined as $L_{\mathrm{NL}}=\left(\bar{\gamma} S_{0}^{(s)}\right)^{-1}$. We apply a fully polarized CW pump beam at the fiber input end along with a polarization-scrambled signal beam. As already pointed out in $[23,24]$, our simulations confirm that, in agreement with the spherical symmetry of Eqs. (3) and (4), all results depend only on the mutual signal-pump SOPs and not on the absolute pump SOP. For definiteness, we fix the pump SOP on the first Stokes axis, (1, 0, 0). The scrambled signal beam is composed by a set of $N$ fully polarized pulses. For each pulse, the ellipticity and azimuthal angle of the field are randomized so as to generate SOPs that are uniformly distributed over the Poincaré sphere, so that the instantaneous DOP (IDOP; i.e., calculated at each point in time),

$$
\operatorname{IDOP}(t)=\frac{\left\|\left\langle\mathbf{S}^{(s)}(t)\right\rangle_{\mathrm{SOP}}\right\|}{S_{0}^{(s)}(t)}
$$

is zero for each $t$ (here the average is taken over all $N$ realizations of the scrambled beams, and it is considered at the fiber input). In Eq. (ㅁ) , we denote by $\|\mathbf{x}\|=\sqrt{\sum_{i=1}^{3} \mathbf{x}_{i}^{2}}$ the Euclidean norm of a vector $\mathbf{x} \in \mathfrak{R}^{3}$. The notation $\langle\cdot\rangle_{\mathrm{SOP}}$ represents statistical averaging, which is performed over the launched signal SOPs.

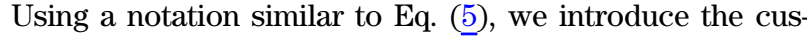
tomary definition of the DOP,

$$
\mathrm{DOP}=\frac{\left\|\left\langle\left\langle\mathbf{S}^{(s)}(t)\right\rangle_{t}\right\rangle_{\mathrm{SOP}}\right\|}{\left\langle S_{0}^{(s)}(t)\right\rangle_{t}}
$$

normalized to the pulse mean power $P_{S}=\left\langle S_{0}^{(s)}(t)\right\rangle_{t}$, which of course does not depend on the launched signal SOP.

Let us emphasize that we considered depolarized signal beams whose fast polarization dynamics vary on the time scale of a few pulses, that is, tens of picoseconds. Hence, each input signal pulse has a constant-in-time SOP (even though the output pulse may have a SOP that is changing across the pulse), and we do not consider the cases when the input pulse has time-varying SOP. Under this assumption, the evaluation of IDOP [Eq. (5)] and DOP [Eq. (ㄷ)] on an ensemble of individual (polarization-scrambled) pulses is in no way restrictive. In fact, the ensemble of scrambled input pulses takes into account all possible input SOPs and therefore is statistically identical to considering a single unpolarized signal beam, whose polarization varies from pulse to pulse. In other words, we assume here that the propagation of polarized pulses in the fiber is an ergodic process; that is, ensemble averaging recovers the same statistics as time averaging. This ergodic property has been demonstrated for counterpropagating NLPs; see [17]. In order to prove that the same property is valid also in the present case of a copropagating NLP, we verified, through numerical simulations, that DOP measurements performed on an ensemble of input pulses, each propagating separately, exactly coincide with those obtained by propagating the pulses in a time sequence, provided that they are sufficiently spaced to avoid pulse-to-pulse interaction mediated by the pump. Actually, the term "sufficiently" can be quantified more accurately: the temporal separation between pulses in the stream should be not less than the total delay time between the signal and the pump channels accumulated over the total length of the fiber plus the pulse duration. For the parameter values used in our simulations (see Section 3), such a total delay is limited to a maximum of $83.2 \mathrm{ps}$, and the effective pulse duration is $12.5 \mathrm{ps}$; hence a pulse period of $100 \mathrm{ps}$ is sufficient so that, if pulses are representative of intensitymodulated bits, the corresponding bit rate is $10 \mathrm{~Gb} / \mathrm{s}$.

After fixing the number of parameters as indicated above, we still remain with three free parameters: the pump power $P_{p}=S_{0}^{(p)}$, the total fiber length $L$, and the amount of walkoff $T_{D}$, which is calculated as the overall delay/advance of the signal pulse with respect to the pump beam, accumulated over the total fiber length $L$. Clearly the total pump-signal delay due to walk-off is the product of channel separation $\Delta \lambda=$ $\lambda_{s}-\lambda_{p}$ times fiber length $L$, times the GVD parameter: $T_{D}=D \Delta \lambda L$, where the GVD parameter $D$ is evaluated at the pump wavelength. In the numerical results of the following sections, for each tested fiber length $L$ we fix the GVD parameter $D=1 \mathrm{ps} / \mathrm{nm} \cdot \mathrm{km}$ and vary the wavelength spacing $\Delta \lambda$ in order to achieve the desired delay $T_{D}$. As an example, for a channel separation $\Delta \lambda=1 \mathrm{~nm}$ and a fiber length of $L=2.6 \mathrm{~km}$, the walk-off $T_{D}$ is $2.6 \mathrm{ps}$, which is almost negligible with respect to the signal-pulse FWHM temporal duration considered here (12.5 ps). However, for $L=20.8 \mathrm{~km}$ and $\Delta \lambda=4 \mathrm{~nm}$, the walk-off becomes as large as $83.2 \mathrm{ps}$. Whenever necessary, by choosing fibers with larger GVD 
parameters we may increase the total pump-signal delay even further. Note that the limit case $T_{D}=0$ may be obtained with group-velocity-matched signals and pumps [e.g., placed on opposite sides of the fiber zero-dispersion wavelength (ZDW)] [2]. Besides this configuration, in general testing walk-off delays close to zero for any specific fiber type with a given dispersion $D$, one should let $\Delta \lambda \simeq 0$, which is not a realistic condition because pump and signal spectra would overlap. Otherwise, one may keep $\Delta \lambda$ fixed to a minimum spacing and decrease $D$, for instance by properly designing the dispersion profile of the fiber. Nevertheless, as far as numerical simulations are concerned, the two approaches (fixed $D$ or fixed $\Delta \lambda$ ) are totally equivalent. In fact, as we verified numerically, for a fixed $L$, our results only depend on the product $D \Delta \lambda$. Hence, it is only the amount of total delay $T_{D}$ that determines the resulting DOP.

Regarding GVD, however, it should be noted that in the presence of a strong Kerr nonlinearity, a large value of $D$ would introduce a considerable pulse distortion due to the interaction between self-phase modulation and chromatic dispersion. To avoid such a destructive effect, fibers with moderate dispersion, such as dispersion-shifted or dispersion-flattened fibers, should be used for the NLP to be effective. Moreover, to ensure that the short signal pulses are not drastically distorted, the results that follow were obtained by placing the signal carrier on the fiber ZDW. However, in order to test the robustness of NLP against moderate amounts of chromatic dispersion, we also performed numerical simulations that confirmed that the NLP is still effective in the presence of nonzero GVD at the signal wavelength. For a $L=20.8 \mathrm{~km}$ long fiber with $D\left(\lambda_{s}\right)=1 \mathrm{ps} / \mathrm{nm} \cdot \mathrm{km}$, with $1 \mathrm{~W}$ signal power, we verified that although the signal pulses are distorted, though not spoiled, the obtained DOP values are similar (i.e., DOP variations within 0.1 ) to the case where GVD is not present.

Another known limit of the mathematical model discussed above is that pump and signal propagate according to their respective equations, as in Eqs. (3) and (4). Each equation is implicitly expressed with respect to its own carrier frequency, thus neglecting the four-wave mixing (FWM) frequency components that arise in the outer signal bandwidths due to nonlinearity. The power of the FWM sidelobes grows at the expense of the interacting pump and signal powers and could eventually degrade the effectiveness of the attraction process. In order to check the impact of FWM and its possible consequences on NLP performance degradation, we complemented the results presented in the following sections with extra numerical simulations, including the effect of FWM. To this purpose, we resorted to a full propagation model that is as general as possible, accounting for all linear and nonlinear effects relevant to the propagation of intense picosecond pulses through telecom fibers. In such a full propagation model, pump and signal are collected in a single multiplexed propagating field with a bandwidth large enough to include FWM sidelobes. Due to such a large bandwidth, these simulations are computationally costly; hence we only performed them for the most critical values of system parameters, namely, a long fiber $(L=20.8 \mathrm{~km})$, large pump powers $\left(P_{p}=1-2 \mathrm{~W}\right)$, and two extreme values of wavelength spacing (narrow, $\Delta \lambda=0.8 \mathrm{~nm}$, or wide, $\Delta \lambda=4.0 \mathrm{~nm}$ ). The other parameters have the same values reported above.
In particular, we consider the chromatic dispersion of $D=$ $1 \mathrm{ps} / \mathrm{nm} \cdot \mathrm{km}$ at both pump and signal wavelengths so that the previouly discussed effect of the GVD-induced pulse broadening is also included. All resulting simulations confirm that the joint impact of FWM and GVD does not alter DOP values by more than 0.1 . In order to further check that power depletion due to FWM is negligible in all tested configurations, we directly measured the FWM sidelobes. These are barely visible in the spectra, with a FWM spectral peak that lies more than $30 \mathrm{~dB}$ below the spectral peak of the pump (and more than $20 \mathrm{~dB}$ below that of the signal). We can then conclude that, at the power levels that we tested, there is no significant degradation of the NLP effectiveness due to FWM.

\section{RESULTS ON ZERO-PMD FIBERS}

Let us begin with the simple case of zero walk-off and evaluate the IDOP, across the signal pulse, as a function of time offset with respect to the pulse peak. The result is shown in Fig. 1(a). The distribution of the IDOP approximately follows the intensity distribution. This is not always the case because a pulse power beyond a certain threshold would cause the IDOP to decrease [2]. The reason for the behavior of the IDOP in Fig. 1(a) is that, for the parameters chosen in this numerical experiment, such a power threshold is equal to $1 \mathrm{~W}$; hence the maximum of the IDOP precisely occurs at the peak pulse intensity (which is equal to $1 \mathrm{~W}$ ). The wings of the pulse are less intensive; therefore the repolarization effect is less effective in the pulse wings until it vanishes completely on the pulse tails. Thus Fig. 1(a) points to the drawback of a copropagating NLP scheme without walk-off: the IDOP quickly degrades away from the pulse peak. On the other hand, the other plots in Fig. 1 include a delay between pump and signal and reveal the role of the walk-off effect in terms of IDOP. In Fig. 1(d) we can see that using a relatively large walk-off $\left(T_{D}=42 \mathrm{ps}\right)$ and pump power $(2 \mathrm{~W})$, along with a longer fiber $(L=10.4 \mathrm{~km})$, brings us closer to an ideal situation: namely, a flat distribution of the IDOP. In fact, the optimal situation would be that of achieving a constant IDOP, as close to unity as possible, across the entire signal pulse.

While the input pulses are characterized by a constant SOP, the IDOP variations across the pulse, as reported in Fig. 1, imply that the SOP of the output pulse is not constant. As was demonstrated in [2] for CW beams, the SOP of the outcoming signal is, on average, attracted toward the SOP of the input pump beam. Therefore, we can define an alignment parameter by averaging the cosine of the angle between the output signal Stokes vectors $\mathbf{S}^{(s)}(L)$ and that of the input pump $\mathbf{S}^{(p)}(0)$, around which the signal is attracted, on average:

$$
I A_{0 L}(t)=\frac{\left\langle\mathbf{S}^{(s)}(t)\right\rangle_{\mathrm{SOP}}}{S_{0}^{(s)}(t)} \cdot \frac{S^{(p)}(0)}{S_{0}^{(p)}(0)},
$$

where $\mathbf{S}^{(s)}(t)=\mathbf{S}^{(s)}(t, L)$ is a shorthand notation for the output-signal Stokes vector. In close analogy to the IDOP definition, Eq. (7) is time dependent; hence it defines an instantaneous alignment parameter for each point across the pulse. In Fig. 1, $I A_{0 L}(t)$ is plotted along with the IDOP and superimposed to it. The two quantities are in fact not distinguishable, at least for the time instants when pulse power is nonnegligible, showing that the signal repolarization 

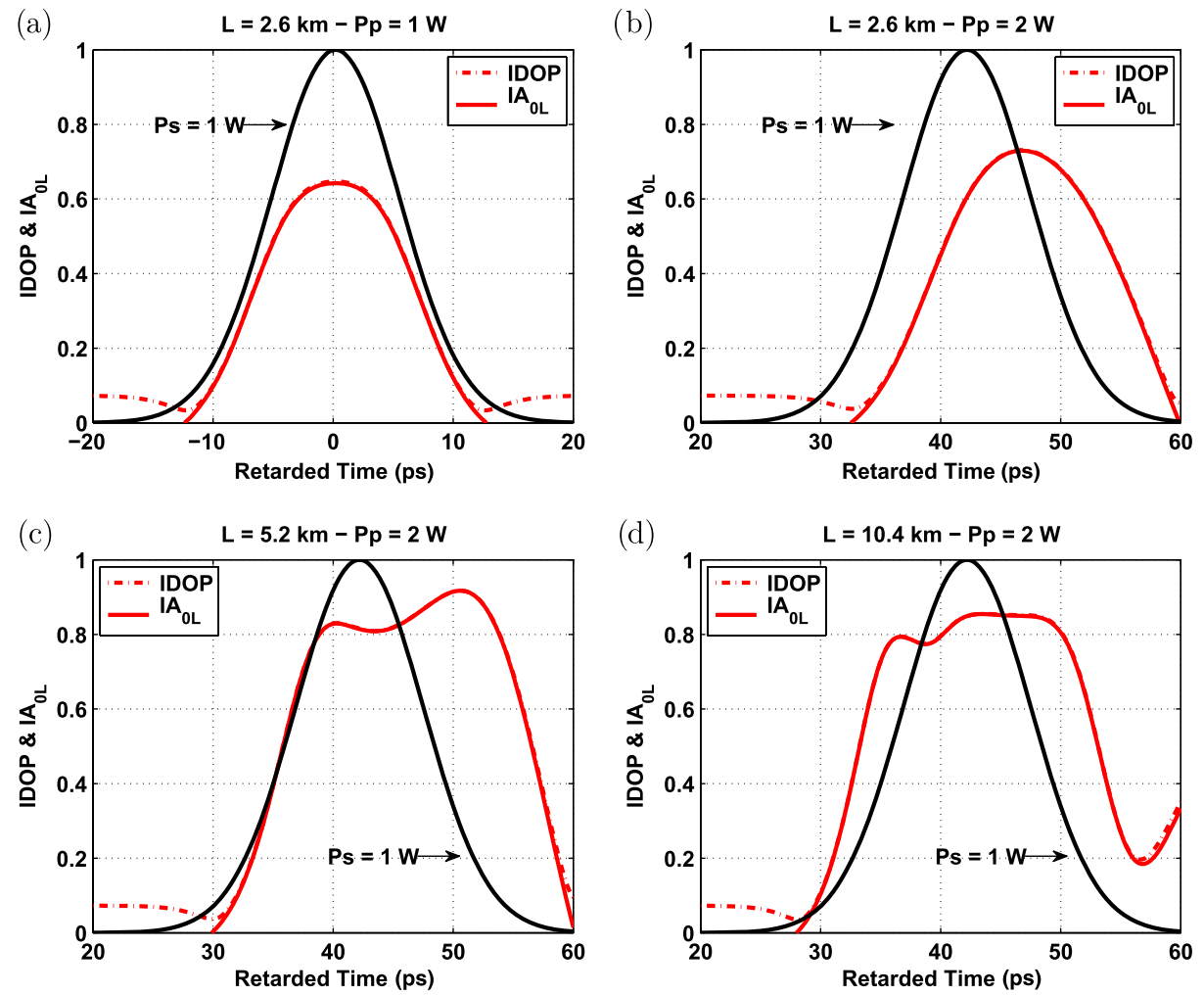

Fig. 1. (Color online) Signal power (solid black), instantaneous DOP (solid red), and instantaneous alignment parameter (dashed red) at the output of the fiber, all versus retarded time: (a) fiber length $L=2.6 \mathrm{~km}$, pump power $P_{p}=1 \mathrm{~W}$, no walk-off; (b) $L=2.6 \mathrm{~km}, P_{p}=2 \mathrm{~W}$, total delay $T_{D}=42 \mathrm{ps}$; (c) $L=5.2 \mathrm{~km}, P_{p}=2 \mathrm{~W}, T_{D}=42 \mathrm{ps}$; and (d) $L=10.4 \mathrm{~km}, P_{p}=2 \mathrm{~W}, T_{D}=42 \mathrm{ps}$.

is associated with the alignment of its SOP with the input pump SOP. We shall further investigate on this point in the following, in a more general framework.

In order to evaluate the performance of the NLP, however, we cannot resort to the time-varying IDOP nor to its peak value, which would largely overestimate the overall pulse repolarization. On the other hand, the progressive flattening of the function $\operatorname{IDOP}(t)$ [and of $I A_{0 L}(t)$ ] in Fig. 1 is the result of walk-off and larger propagation lengths. To get a physical understanding of this phenomenon, we should consider that, thanks to the walk-off, the signal pulse continuously meets "fresh" portions of the pump beam during propagation, and it interacts with them over longer distances. This way, even the weak tails of the pulse start to experience significant nonlinearly induced repolarization, which in turn increases the overall DOP of the signal.

Indeed, the appropriate metric to quantify the NLP performance for the pulsed signal beam is the conventional definition of DOP [Eq. (6)], which involves a double averaging-one over time and the other over the ensemble of scrambled beams. Figure 2 shows the DOP as a function of the total signal-pump delay, induced by walk-off, for various values of pump power and fiber length. For the range of delays tested in Figs. 2(a) through 2(d), our results confirm the conclusion that was qualitatively drawn above; that is, except for the case of a short fiber, which is impractical because of its very poor performance, the larger the walk-off, the higher the DOP. With the purpose of comparison, we note that the best DOP for the case of no walk-off is of only 0.49 [see Fig. 2(a)], while the inclusion of walk-off may improve this result up to 0.83 [see Fig. 2(d)] (the DOP becomes as large as 0.9 if losses are neglected). Most likely, even such a high value is not the limit, and higher values can be achieved in a fully optimized configuration. However, the optimization of the present NLP scheme is beyond the scope of this paper.

In analogy with the DOP, it is desirable to introduce a single alignment parameter instead of a continuous function $I A_{0 L}(t)$, which is able to summarize the angular distance between the attracting pump SOP and the output, time-varying, signal SOP:

$$
A_{0 L}=\frac{\left\langle\left\langle\mathbf{S}^{(s)}(t)\right\rangle_{t}\right\rangle_{\mathrm{SOP}}}{\left\langle S_{0}^{(s)}(t)\right\rangle_{t}} \cdot \frac{S^{(p)}(0)}{S_{0}^{(p)}(0)} .
$$

In Fig. 2 we also plot the alignment parameter [Eq. (ㅁ)] along with the DOP. Similar to Fig. 1, the two quantities are superimposed, further confirming that the repolarization of the signal beam is indeed associated with the alignment of its SOP along the input SOP of the pump beam. We shall discuss this issue in more detail in Section 4, where we will show that the presence of PMD breaks the coincidence of DOP and $I A_{0 L}(t)$.

\section{A. Repolarization with an Incoherent Pump Beam}

Frequently in practice the power level of the pump beam fluctuates around some average value rather than remaining constant in time, as was supposed in our previous considerations. Moreover, such power fluctuations are often introduced on purpose. As a matter of fact, stimulated Brillouin scattering (SBS), which is the lowest-threshold nonlinear effect for $\mathrm{CW}$ beams propagating in optical fibers, would introduce a significant pump depletion that prevents the injection of 
(a)

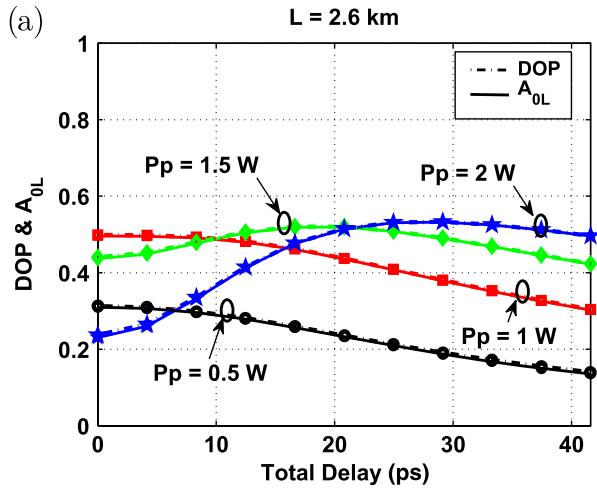

(c)

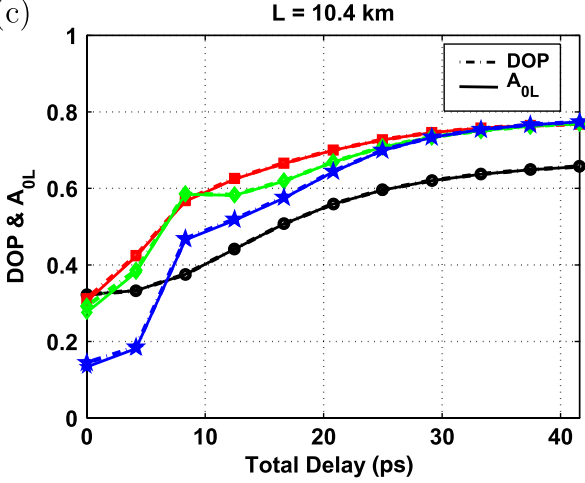

(b)

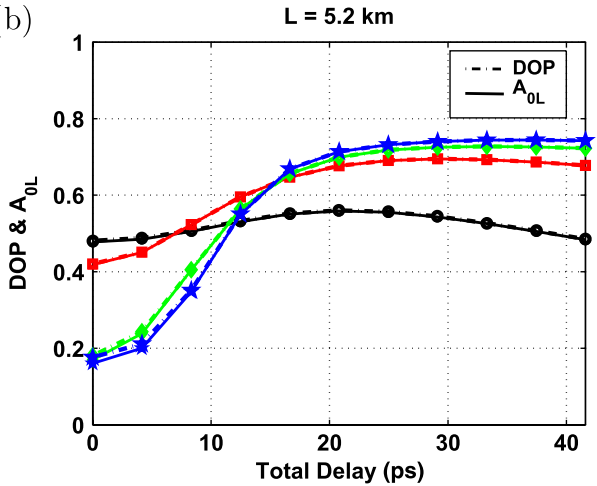

(d)

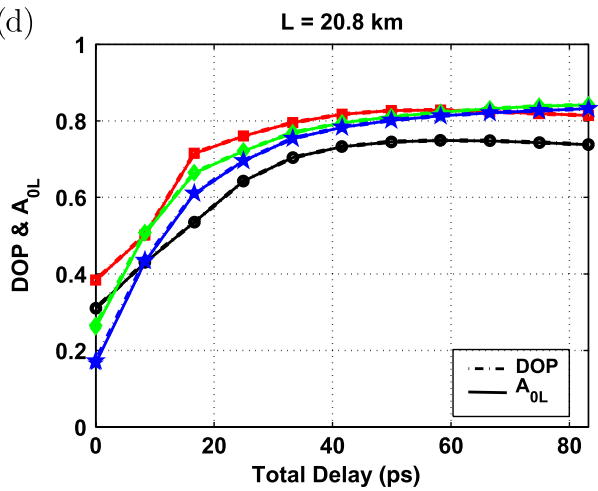

Fig. 2. (Color online) Degree of polarization (dashed curves) and alignment parameter (solid curves) as a function of total delay in the case of fibers without PMD. Results are plotted for fiber lengths $L=2.6,5.2,10.4$, and $20.8 \mathrm{~km}$ in (a)-(d), respectively, and different pump powers $P_{p}=0.5$, 1, 1.5, and $2 \mathrm{~W}$ (identified by the following symbols, respectively: circle, square, diamond, star). Note that solid and dashed curves coincide.

watt-level CW pump beams in an NLP. The standard method to suppress SBS is that of broadening the spectrum of a CW pump by means of a gigahertz-rate phase modulation. A simpler and cheaper solution for the suppression of SBS, which was implemented for counterpropagating NLPs in [8], is that of using an incoherent pump beam. A fully polarized incoherent beam is characterized by large amplitude (intensity) fluctuations. In order to model such an incoherent pump beam in our setup, it is sufficient to extend our analysis from the case of a coherent and stable intensity beam to an ensemble of coherent beams that experience large intensity fluctuations. In our simulations for the case of no walk-off we have assumed that the amplitude of each polarization component of the pump beam is a stochastic Gaussian process with zero mean and standard deviation, chosen in such a way that the time-average of the pump intensity yields $0.25,0.5$, or $1 \mathrm{~W}$. After performing an average over 100 realizations, we obtained the plot shown in Fig. $\underline{3}$. When compared to the case of a coherent pump beam, the performance of the NLP driven by an incoherent pump beam only shows a slight degradation (DOP $=0.5$ against 0.73 ). Nevertheless, an efficient repolarization effect is still clear; moreover, the repolarization remains particularly strong whenever the walk-off is large. The observed DOP degradation clearly arises from the dips and spikes in the pump intensity, which are not desirable because the NLP is a nonlinear device whose performance critically depends on the level of the pump intensity. At the same time, larger amounts of walk-off correspond to a faster sliding of the signal pulse throughout the (CW) pump. Thus, the signal pulse slides through a larger number of dips and spikes of the incoherent pump, and on average it sees a smoothened intensity profile of the pump beam. For this reason, the DOP values in Fig. 3 corresponding to the largest total delays (around $80 \mathrm{ps}$ ) are very close to those obtained in the case of a coherent pump with stable intensity [see Fig. 3(d)] for the same average power. Note that we use a sufficiently broadband ( $600 \mathrm{GHz}$ wide) pump beam and allow it to interact with the signal pulse over a sufficiently long distance $(L=20.8 \mathrm{~km}$ in our example). The broadband nature of the pump beam is necessary for the goal of preventing the signal pulse from resolving individual temporal amplitude variations of the pump beam. In other words, the signal pulse should effectively "see" only the average level of the pump intensity. Otherwise, the smooth nature of the intrapulse signal SOP will not be preserved across a single pulse and will change from one realization to another.

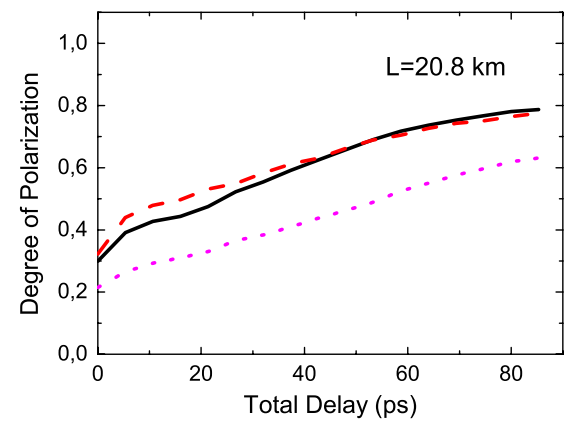

Fig. 3. (Color online) DOP of the NLP driven by incoherent ("noisy") pump with mean power $\left\langle P_{p}\right\rangle_{t}: 0.25 \mathrm{~W}$ (magenta dotted curve), $0.5 \mathrm{~W}$ (black solid curve), and $1 \mathrm{~W}$ (red dashed curve). Fiber losses are not taken into account. 


\section{PMD-INDUCED DEGRADATION OF THE PERFORMANCE OF NLPS}

We wish now to extend our study of the NLP to the case of fibers where PMD is present. Standard telecom fibers are characterized by random birefringence, which, as recalled in Section 2, depends on the optical frequency through the PMD coefficient $D_{p}$, which is typically expressed in $\mathrm{ps} / \sqrt{\mathrm{km}}$. With a single propagating field, the primary effect of PMD is to introduce a stochastic delay, which depends on the SOP of the launched field and whose range is quantified by the differential group delay $\Delta \tau$ [25]. This is in turn a random quantity, with Maxwellian distribution, whose root mean square value $\Delta \tau_{\text {rms }}=D_{p} \sqrt{L}$ depends on the fiber length and the PMD coefficient. Although such an effect can be negligible for pulses whose duration is much larger than $\Delta \tau_{\mathrm{rms}}$, another nonnegligible effect of PMD occurs in a two-channel (pump-signal) scenario such as the one that we are studying here. Indeed, the polarization of optical beams propagating at different wavelengths is subject to different amounts of birefringence. Hence, when two beams are launched into the fiber, the mutual position of their initial SOPs is not maintained along the fiber and the SOPs eventually diffuse on the Poincaré sphere. This effect occurs on a length scale that is characterized by the diffusion length $L_{d}$, whose expression is given in Section 2.

As discussed in Section 3, signal repolarization occurs around the SOP of the pump beam. For an effective performance of the NLP, it is thus necessary that, despite PMD, pump and signal polarizations evolve in the same way along the fiber. This only happens if $D_{p}$ and the pump-signal wavelength spacing are sufficiently small so that $L_{d}$ is much larger than the fiber length where the NLP effect takes place. From a physical standpoint, it is the mutual pump and probe SOP evolution that determines the difference between the operation of the NLP in the diffusion limit or in the Manakov limit.

The results reported in Fig. 2 show the DOP and the alignment parameter $A_{0 L}$, which were defined in Section 3. However, in the case of fibers with PMD, the definition of these two parameters needs a further specification. The statistical averaging $\langle\cdot\rangle_{\mathrm{SOP}}$ in the definition of DOP [Eq. (6)] is performed over the launched signal SOPs, which are uniformly scattered over the Poincare sphere at the input and are eventually attracted closer to an attraction $S O P$ at the output of the NLP. In the presence of PMD, such an attraction SOP may not coincide with the input pump SOP, yet the attraction SOP may be obviously be evaluated $a$ posteriori by averaging the output signal SOPs. Let us define $\mathbf{S}^{(a)}=P_{s}^{-1}\left\langle\left\langle\mathbf{S}^{(s)}(t)\right\rangle_{t}\right\rangle_{\mathrm{SOP}}$, which is the vector appearing in the definition [Eq. (8)] of $A_{0 L}$, and whose magnitude is the DOP [Eq. (6)]. The attraction SOP is indeed a unit-magnitude vector that is parallel to $\mathbf{S}^{(a)}$ and that represents a "center of mass" for the ensemble of output SOPs, which are obtained for the different input launched pulses. Because PMD is a stochastic phenomenon, the direction of $\mathbf{S}^{(a)}$ is expected to change for each fiber realization, although, as PMD is isotropically random, its expectation over the ensemble of fibers (which is here denoted by $\langle\cdot\rangle_{\mathrm{PMD}}$ ) will be parallel to the input pump SOP: $\left\langle\mathbf{S}^{(a)}\right\rangle_{\mathrm{PMD}} \| S^{(p)}(0) / S_{0}^{(p)}(0)$. Moreover, the alignment parameter was defined in Eq. (8), for the case of no PMD, as $A_{0 L}=\mathbf{S}^{(a)} \cdot\left(S^{(p)}(0) / S_{0}^{(p)}(0)\right)$, where the scalar product involves the input pump SOP, whereas $\mathbf{S}^{(a)}$ is evaluated at the fiber output and, as we have pointed out, is subject to change in the presence of PMD. Hence, we shall extend the definition of $A_{0 L}$ to the case of fibers with PMD through a third level of averaging, over fiber realizations, as

$$
\begin{aligned}
A_{0 L}^{\mathrm{PMD}} & =\frac{\left\langle\left\langle\left\langle\mathbf{S}^{(s)}(t)\right\rangle_{t}\right\rangle_{\mathrm{SOP}}\right\rangle_{\mathrm{PMD}}}{\left\langle S_{0}^{(s)}(t)\right\rangle_{t}} \cdot \frac{S^{(p)}(0)}{S_{0}^{(p)}(0)} \\
& =P_{S}^{-1}\left\|\left\langle\left\langle\left\langle\mathbf{S}^{(s)}(t)\right\rangle_{t}\right\rangle_{\mathrm{SOP}}\right\rangle_{\mathrm{PMD}}\right\|,
\end{aligned}
$$

where the last expression stems from the previously discussed parallelism condition. The mean pulse power $P_{S}$ is not subject to change with the launched signal SOP or with the fiber PMD (PMD is a conservative phenomenon); hence it is always treated as a constant when applying the averages $\langle\cdot\rangle_{\mathrm{SOP}}$ and $\langle\cdot\rangle_{\mathrm{PMD}}$. Similarly, to evaluate the average performance of the NLP in terms of DOP, we shall average the DOP, defined in Eq. (6) for a single fiber sample, over the ensemble of fibers with PMD:

$$
\mathrm{DOP}^{\mathrm{PMD}}=P_{S}^{-1}\left\langle\left\|\left\langle\left\langle\mathbf{S}^{(s)}(t)\right\rangle_{t}\right\rangle_{\mathrm{SOP}}\right\|\right\rangle_{\mathrm{PMD}} .
$$

The alignment parameter and the DOP, defined as in Eqs. (9) and (10), are plotted in Figs. 4 and 5 for different fiber lengths and pump powers, whose values are the same as in Fig. 2, and for different randomly birefringent fibers characterized by either a "small" PMD coefficient, $D_{p}=$ $0.05 \mathrm{ps} / \sqrt{\mathrm{km}}$, or a "large" PMD coefficient, $D_{p}=$ $0.2 \mathrm{ps} / \sqrt{\mathrm{km}}$, respectively. The first value is typical of lowPMD fibers manufactured in recent times, that is, after 1999, as reported by the extensive field measurements in [26], while older fibers are characterized by larger $D_{p}$ values, around $0.13 \mathrm{ps} / \sqrt{\mathrm{km}}$ for fibers installed in the mid-1990s and above $0.3 \mathrm{ps} / \sqrt{\mathrm{km}}$ for those installed in the $1980 \mathrm{~s}$. We thus chose the two values above for the PMD coefficient as representative of either modern or legacy fibers.

When comparing Figs. $\underline{2}$ and $\underline{4}$, we barely see any difference, the basic reason being that $D_{p}$ is sufficiently small to guarantee an almost identical evolution of pump and signal polarizations along the fiber, as happens in fibers without PMD. This fact is numerically justified by the values of the diffusion length: the ratio $L_{d} / L$ is between 47 and 187 for the cases tested in Fig. 4 . Hence $L_{d} \gg L$ for each of the tested fiber lengths. On the other hand, when comparing results in Fig. 5 with those in Fig. 4, we can see that the NLP performance, in terms of DOP, is roughly the same in the case of shorter fibers (2.6 and $5.2 \mathrm{~km}$ ), slightly degrades for $10.4 \mathrm{~km}$ long fibers, and is definitely spoiled (i.e., decreased by 0.25 ) by PMD in the best cases of repolarization, namely those of a $20.8 \mathrm{~km}$ long fiber with a large walk-off delay. One thing to note in Fig. 5, when compared with Fig. 2, is that here DOP and $A_{0 L}$ do not coincide any more: a slight mismatch of these two parameters can be observed also in the case of small (but nonzero) PMD in Fig. $\underline{4}$, especially on the right side of the plots. We shall discuss shortly hereafter the reason for this behavior.

Figure 6 compares the output SOPs that are obtained for 50 launched pulses with their initial SOPs uniform on the Poincaré sphere after an NLP composed of a single randomly birefringent fiber sample, in the case of no PMD (left) or with a large PMD coefficient $D_{p}=0.2 \mathrm{ps} / \sqrt{\mathrm{km}}$ (right). The pump 

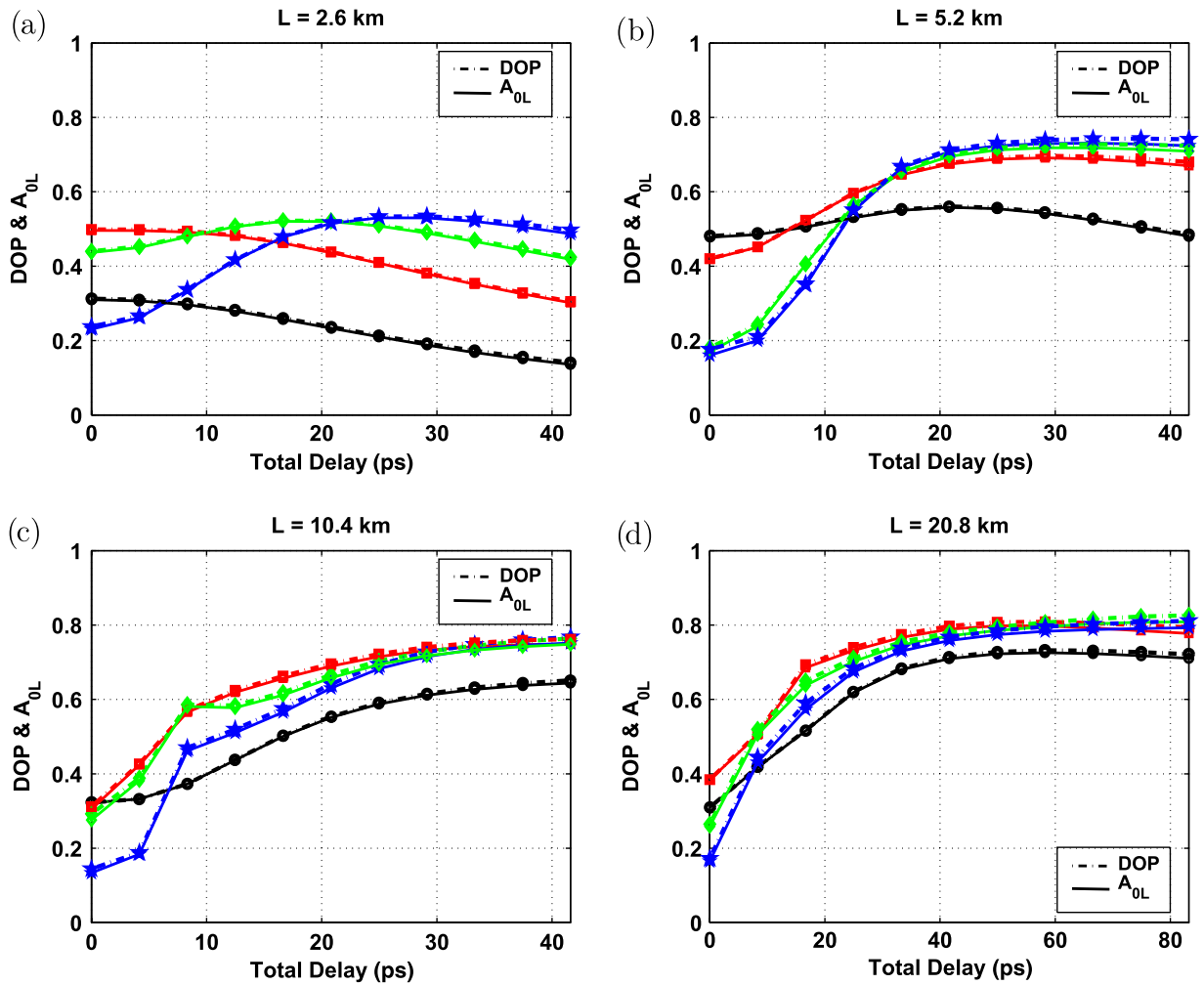

Fig. 4. (Color online) Degree of polarization (dashed curves) and alignment parameter (solid curves) as a function of total delay in the case of modern fibers with a small PMD coefficient $D_{p}=0.05 \mathrm{ps} / \mathrm{km}^{0.5}$. Results are plotted for fiber lengths $L=2.6,5.2,10.4$, and $20.8 \mathrm{~km}$ in (a)-(d), respectively, and different pump powers $P_{p}=0.5,1,1.5$, and $2 \mathrm{~W}$ (identified by the following symbols, respectively: circle, square, diamond, star).

power $P_{p}=2 \mathrm{~W}$, the fiber length $L=20.8 \mathrm{~km}$, and the walkoff delay is $83 \mathrm{ps}$; hence the operating conditions are those on the right edge of the curves with star symbols (blue color) in Figs. 2(d) (no PMD) and 5(d) (large PMD), respectively. The pump SOP is launched on $\overline{S_{1}}$, which is reported as a dark blue triangle in Fig. $\underline{6}$. The linear birefringence of the fiber has been equalized before plotting Fig. $\underline{6}$ so that output signal SOPs are plotted in the input frame of reference. Hence the difference between the input pump SOP $\left(S_{1}\right)$ and the attraction SOP $\mathbf{S}^{(a)}(k) /\left\|\mathbf{S}^{(a)}(k)\right\|$, which is reported as a black square in Fig. 6 , can be appreciated. The difference between the two plots in Fig. 6 is a graphical representation of the reason why the output signal DOP and $A_{0 L}$ may not coincide. In fact, it is not difficult to prove that $A_{0 L}$ and DOP only coincide whenever the attraction SOP is constantly parallel to the pump SOP, for any fiber realization, as is obviously the case of Fig. 6 (left), where PMD is absent. Let us denote by $\mathbf{S}^{(a)}(k)$ the evaluation of $\mathbf{S}^{(a)}=P_{S}^{-1}\left\langle\left\langle\mathbf{S}^{(s)}(t)\right\rangle_{t}\right\rangle_{\mathrm{SOP}}$, that is, the normalized average output signal SOP, conditioned on the $k$ th fiber realization [the direction of $\mathbf{S}^{(a)}(k)$ is the black square in Fig. 6 for the tested fiber realization]. With this specification, Eqs. (9) and (10) can be rewritten as

$$
\mathrm{DOP}=\left\langle\left\|\mathbf{S}^{(a)}(k)\right\|\right\rangle_{\mathrm{PMD}}
$$

and

$$
A_{0 L}=\left\langle\mathbf{S}^{(a)}(k) \cdot \frac{S^{(p)}(0)}{S_{0}^{(p)}(0)}\right\rangle_{\mathrm{PMD}}=\left\langle\left\|\mathbf{S}^{(a)}(k)\right\| \cos \left(\theta_{k}\right)\right\rangle_{\mathrm{PMD}},
$$

where $\cos \left(\theta_{k}\right)=\left(\mathbf{S}^{(a)}(k) /\left\|\mathbf{S}^{(a)}(k)\right\|\right) \cdot\left(S^{(p)}(0) / S_{0}^{(p)}(0)\right)$ quantifies the angular mismatch between the attraction SOP and the pump SOP (i.e., two unit-magnitude vectors), which is visible in Fig. $\underline{6}$ (right). By comparing Eqs. (11) and (12), we may deduce that always $A_{0 L} \leq \mathrm{DOP}$ and that the equal sign requires that $\theta_{k}=0$ for any $k$, that is, that the signal is always attracted toward the pump SOP in spite of the fiber birefringence.

This is what happens in Figs. 2 through $\underline{4}$, with no or little PMD, while in Fig. 5 always $A_{0 L}<$ DOP for a given power level and fiber length. This is especially true for larger walk-off delays, where a larger wavelength spacing between pump and signal brings about a stronger depolarization due to PMD, as is quantified by a shorter diffusion length: the ratio $L_{d} / L$ is in fact between 3 and 12 for the cases tested in Fig. $\underline{5}$. These values are much lower than those reported above in the case of low-PMD fibers $\left(D_{p}=0.05 \mathrm{ps} / \sqrt{\mathrm{km}}\right)$ and do not guarantee that the NLP operates in the Manakov limit, $L_{d} \gg L$, for each of the tested configurations.

For the evaluation of the DOP and $A_{0 L}$, which are reported in Figs. 2, 4, and 5, the averaging in Eqs. (11) and (12) was numerically evaluated by using 50 random input signal SOPs and 10 random fiber samples (the evaluation of $\langle\cdot\rangle_{t}$ is a simple time averaging and is not of a stochastic nature; hence it is performed by time integration on a large number of samples [1024] and does not entail any Monte Carlo sampling) for a total of 500 signal propagations for each tested combination of fiber length, power level, and walk-off delay. Although one would need thousands of fiber samples to properly statistically characterize a pool of randomly birefringent fibers, to limit simulation time to reasonable levels we only tested 10 fibers. However, we also report here the standard deviation $\sigma_{\text {DOP }}$ of the DOP values that were obtained from these fiber samples. For $2.6 \mathrm{~km}$ long fibers, whose mean DOP is reported 

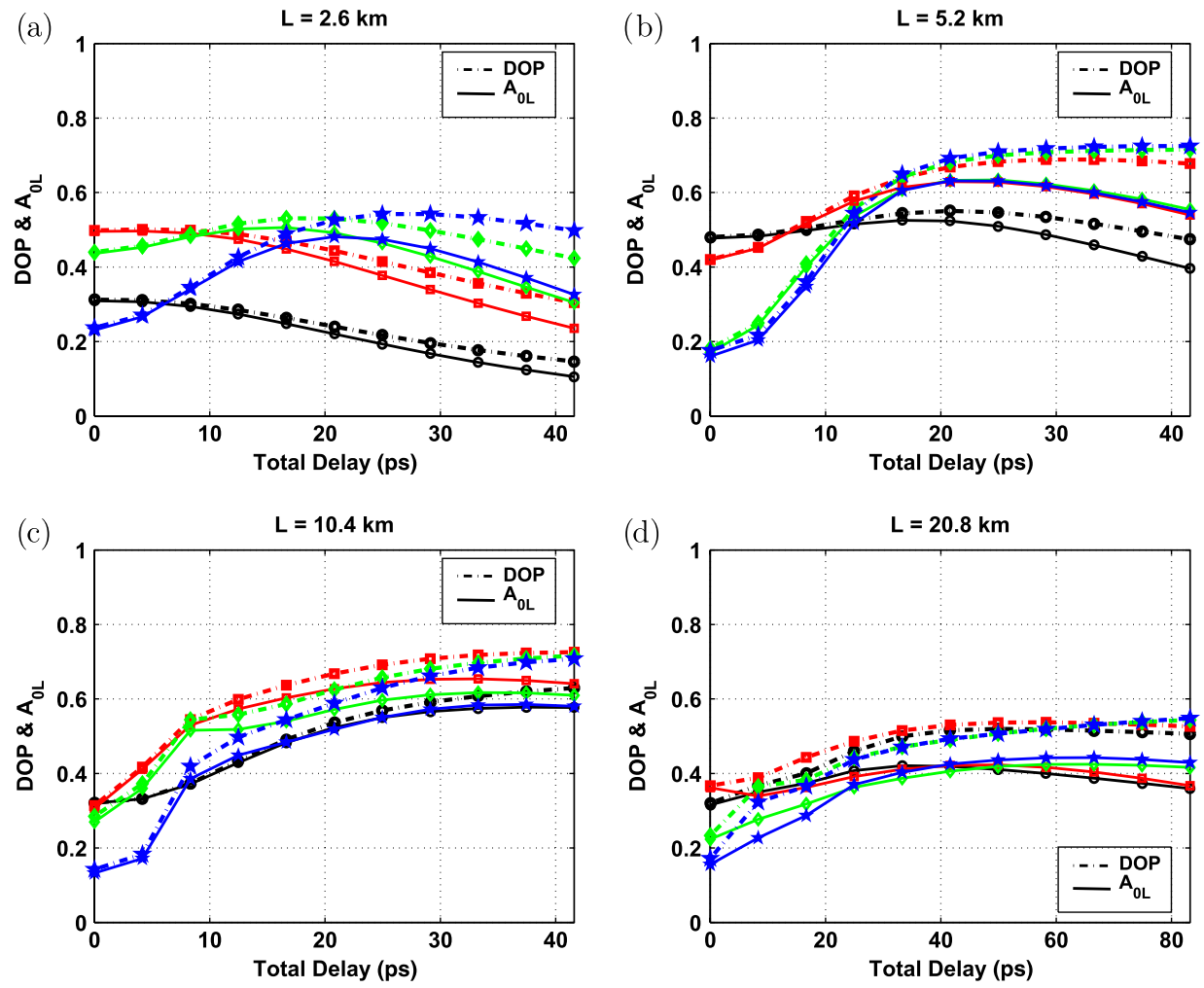

Fig. 5. (Color online) Degree of polarization (dashed curves) and alignment parameter (solid curves) as a function of total delay in the case of legacy fibers with a large PMD coefficient $D_{p}=0.2 \mathrm{ps} / \sqrt{\mathrm{km}}$. Results are plotted for fiber lengths $L=2.6,5.2,10.4$, and $20.8 \mathrm{~km}$ in (a)-(d), respectively, and different pump powers $P_{p}=0.5,1,1.5$, and $2 \mathrm{~W}$ (identified by the following symbols, respectively: circle, square, diamond, star).
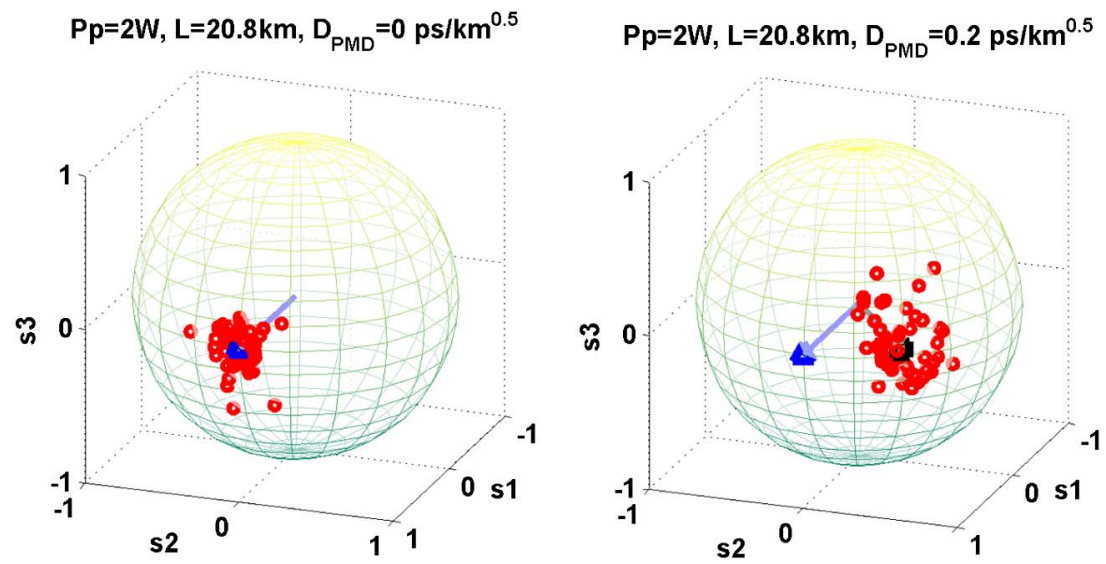

Fig. 6. (Color online) Distribution of 50 (initially) random signal SOPs after the NLP with a $20.8 \mathrm{~km}$ long fiber, with the pump power $P_{p}=2 \mathrm{~W}$ and the input pump SOP on $\hat{S}_{1}$ (see plots). Without PMD (left), the SOPs surround the pump SOP, which acts as the attraction SOP. With large PMD (right), the attraction SOP $\mathbf{S}^{(a)}(k) /\left\|\mathbf{S}^{(a)}(k)\right\|$ no longer coincides with the pump SOP.

in Fig. $5(\mathrm{a})$, the $\sigma_{\mathrm{DOP}}$ is below 0.04 , whereas the standard deviation strongly increases in the case of longer $20.8 \mathrm{~km}$ fibers until it reaches the highest value $\left(\sigma_{\mathrm{DOP}}=0.24\right)$ for the largest walk-off delays. Hence, the DOP reported in Fig. 5(d) is the mean of fiber samples that exhibit strong statistical oscillations that depend on the specific sample, as is typically the case in the presence of PMD. On the contrary, fibers with a small PMD coefficient are subject to little variability and induce much smaller fluctuations on the obtained DOP values: in the case $D_{p}=0.05 \mathrm{ps} / \sqrt{\mathrm{km}}$, the mean DOP is plotted in Fig. 4 and $\sigma_{\mathrm{DOP}}$ is at most of the order of 0.01 for all tested fiber lengths. The standard deviation of the DOP values is of course zero in the case of Fig. 2, where PMD is not present.

\section{CONCLUSION}

In this work we have quantified the performance of an NLP based on interaction of a copropagating CW pump beam with a signal beam consisting of a sequence of short pulses (12.5 ps FWHM) transmitted at a pulse rate of $10 \mathrm{GHz}$. Both beams are supposed to be strong enough to induce a sufficient 
cross-polarization interaction over a span of fiber of a few kilometers. Our main objective was the comparison of the performance of the NLP with two beams in the absence of the walk-off, on the one hand, and the NLP with the effect of the walk-off included, on the other hand. A remarkable result is the finding that the latter performs much better, provided that the walk-off exceeds several pulse widths and the fiber accommodates many $(\sim 5-10)$ nonlinear lengths.

Indeed, from the results of Figs. $\underline{2}$ through $\underline{5}$, some general conclusions can be drawn on the effectiveness of the NLP in the presence of pump-signal walk-off. Whenever the total delay due to walk-off is sufficiently large, a larger pump power always yields a better DOP, whereas this is not true in the absence of or with a limited walk-off, that is, below two pulse periods. The presence of walk-off due to chromatic dispersion is in most cases helpful for increasing the NLP performance, especially for longer fibers or larger pump powers. An optimization of the walk-off delay depending on other parameters, such as length, power, and PMD, is beyond the scope of this paper and is left to future research.

Another important objective of our study was the numerical evaluation, by means of Monte Carlo simulations, of the impact of PMD on the performance of the NLP whenever a randomly birefringent telecom fiber is employed for the repolarization process. The presence of a small amount of PMD, such as that brought about by recently manufactured fibers, with a PMD coefficient $D_{p}=0.05 \mathrm{ps} / \sqrt{\mathrm{km}}$, does not substantially alter the performance of the NLP and is well tolerated even in the longer $(L=20.8 \mathrm{~km})$ tested fiber case. On the other hand a further increase of PMD, for instance due to the use of legacy fibers with a relatively large PMD coefficient, such as $D_{p}=0.2 \mathrm{ps} / \sqrt{\mathrm{km}}$, causes a degradation of the NLP performance. Such degradation is especially clear in the case of longer fibers and large walk-off delays, where the best DOP values are achieved. The reason for the degradation is that, in such operating conditions, the polarization of the pump, as seen by the signal SOP, is randomly wandering along the fiber due to PMD; hence the pump SOP is not sufficiently stable to attract the signal SOP. Such a situation, which we called the diffusion limit, was numerically characterized by considering the system parameters such that the diffusion length $L_{d}$ and fiber length $L$ tend to approach each other.

As far as the practical implementation of the NLP for optical-fiber telecommunications systems is concerned, a drawback is provided by the relatively high power levels that are required to induce a sufficient cross-polarization interaction. For applications involving an NLP at the receiver side, signals may be amplified up to the necessary power levels by an erbium-doped fiber amplifier without introducing nonlinear impairments in the transmission line. Moreover, as was shown in [27] for a counterpropagating NLP, the DOP value depends on the product of signal and pump powers. Hence it should be possible to employ unbalanced power levels, as also done in [18], where a powerful pump repolarizes a relatively weak signal. Such a solution for the present copropagating geometry deserves further investigation.

\section{ACKNOWLEDGMENTS}

This work was carried out with support from the Programma di Ricerca Scientifica di Rilevante Interesse Nazionale 2008 project "Nonlinear cross-polarization interactions in photonic devices and systems.”

\section{REFERENCES}

1. J. E. Heebner, R. S. Bennink, R. W. Boyd, and R. A. Fisher, "Conversion of unpolarized light to polarized light with greater than $50 \%$ efficiency by photorefractive two-beam coupling," Opt. Lett. 25, 257-259 (2000).

2. V. V. Kozlov, K. Turitsyn, and S. Wabnitz, "Nonlinear repolarization in optical fibers: polarization attraction with copropagating beams," Opt. Lett. 36, 4050-4052 (2011).

3. S. Pitois, G. Millot, and S. Wabnitz, "Polarization domain wall solitons with counterpropagating laser beams," Phys. Rev. Lett. 81, 1409-1412 (1998).

4. S. Pitois, G. Millot, and S. Wabnitz, "Nonlinear polarization dynamics of counterpropagating waves in an isotropic optical fiber: theory and experiments," J. Opt. Soc. Am. B 18, 432-443 (2001)

5. S. Pitois, A. Sauter, and G. Millot, "Simultaneous achievement of polarization attraction and Raman amplification in isotropic optical fibers," Opt. Lett. 29, 599-601 (2004).

6. S. Pitois, J. Fatome, and G. Millot, "Polarization attraction using counter-propagating waves in optical fiber at telecommunication wavelengths," Opt. Express 16, 6646-6651 (2008).

7. S. Pitois, A. Picozzi, G. Millot, H. R. Jauslin, and M. Haelterman, "Polarization and modal attractors in conservative counterpropagating four-wave interaction," Europhys. Lett. 70, 88-94 (2005).

8. J. Fatome, S. Pitois, P. Morin, and G. Millot, "Observation of light-by-light polarization control and stabilization in optical fibre for telecommunication applications," Opt. Express 18, 15311-15317 (2010).

9. P. Morin, J. Fatome, C. Finot, S. Pitois, R. Claveau, and G. Millot, "All-optical nonlinear processing of both polarization state and intensity profile for $40 \mathrm{Gbit} / \mathrm{s}$ regeneration applications," Opt. Express 19, 17158-17166 (2011)

10. D. Sugny, A. Picozzi, S. Lagrange, and H. R. Jauslin, "Role of singular tori in the dynamics of spatiotemporal nonlinear wave systems," Phys. Rev. Lett. 103, 034102 (2009).

11. E. Assémat, S. Lagrange, A. Picozzi, H. R. Jauslin, and D. Sugny, "Complete nonlinear polarization control in an optical fiber system," Opt. Lett. 35, 2025 (2010).

12. S. Lagrange, D. Sugny, A. Picozzi, and H. R. Jauslin, "Singular tori as attractors of four-wave-interaction systems," Phys. Rev. E 81, 016202 (2010).

13. E. Assémat, S. Lagrange, A. Picozzi, H. R. Jauslin, and D. Sugny, "Complete nonlinear polarization control in an optical fiber system," Opt. Lett. 35, 2025-2027 (2010).

14. V. V. Kozlov, J. Nuño, and S. Wabnitz, "Theory of lossless polarization attraction in telecommunication fibers," J. Opt. Soc. Am. B 28, 100-108 (2011).

15. E. Assemat, D. Dargent, A. Picozzi, H. R. Jauslin, and D. Sugny, "Polarization control in spun and telecommunication optical fibers," Opt. Lett. 36, 4038-4040 (2011).

16. V. V. Kozlov and S. Wabnitz, "Theoretical study of polarization attraction in high-birefringence and spun fibers," Opt. Lett. 35, 3949-3951 (2010).

17. V. V. Kozlov, J. Fatome, P. Morin, S. Pitois, G. Millot, and S. Wabnitz, "Nonlinear repolarization dynamics in optical fibers: transient polarization attraction," J. Opt. Soc. Am. B 28, 1782-1791 (2011).

18. S. Pitois and M. Haelterman, "Optical fiber polarization funnel," in Nonlinear Guided Waves and Their Applications, OSA Technical Digest Series (Optical Society of America, 2001), pp. 278-280.

19. V. V. Kozlov, Javier Nuño, J. D. Ania-Castañón, and S. Wabnitz, "Theory of fiber optics Raman polarizer," Opt. Lett. 35, 3970-3972 (2010).

20. S. V. Manakov, "On the theory of two-dimensional stationary self focussing of electromagnetic waves," Sov. Phys. J. Exper. Theor. Phys. 38, 248-253 (1974).

21. D. Marcuse, C. R. Menyuk, and P. K. A. Wai, "Application of the Manakov-PMD equation to studies of signal propagation in optical fibers with randomly varying birefringence," J. Lightwave Technol. 15, 1735-1746 (1997). 
22. P. Serena, M. Bertolini, and A. Vannucci, "Optilux Toolbox," http://optilux.sourceforge.net/Documentation/optilux_doc.pdf.

23. M. Barozzi, A. Vannucci, and D. Sperti, "A simple counterpropagation algorithm for optical signals (SCAOS) to simulate polarization attraction," in Proceedings of Fotonica 2012 (AEIT - Federazione Italiana di Elettrotecnica, Elettronica, Automazione, Informatica e Telecomunicazioni, 2012), A6.4.

24. M. Barozzi, A. Vannucci, and D. Sperti, "Lossless polarization attraction simulation with a novel and simple counterpropagation algorithm for optical signals," JEOS RP 7, 12042 (2012).
25. A. Bononi and A. Vannucci, "Is there life beyond the principal states of polarization?," Opt. Fiber Technol. 8, 257-294 (2002).

26. D. Breuer, H.-J. Tessmann, A. Gladisch, H. M. Foisel, G. Neumann, H. Reiner, and H. Cremer, "Measurements of PMD in the installed fiber plant of Deutsche Telekom," in IEEE LEOS Summer Topical Meetings (IEEE, 2003), pp. MB2.1-MB2.2.

27. M. Barozzi and A. Vannucci, "Performance analysis of lossless polarization attractors," in Latin America Optics and Photonics Conference, OSA Technical Digest (online) (Optical Society of America, 2012), paper LM3C.4. 